\title{
Expression of leptin receptor in endometrial biopsies of endometrial and ovarian cancer patients
}

\author{
LUIS FERNANDO MÉNDEZ-LÓPEZ ${ }^{1,2}$, MARTHA IMELDA DÁVILA-RODRÍGUEZ ${ }^{2}$, ANGEL ZAVALA-POMPA ${ }^{3}$, \\ ERNESTO TORRES-LÓPEZ ${ }^{4}$, BLANCA EDELIA GONZÁLEZ-MARTÍNEZ ${ }^{1}$ \\ and MANUEL LÓPEZ-CABANILLAS-LOMELÍ ${ }^{1}$
${ }^{1}$ Faculty of Public Health and Nutrition, Autonomous University of Nuevo León, Monterrey, Nuevo León 64460;
${ }^{2}$ Division of Genetics, Northeast Biomedical Research Center, Mexican Social Security Institute, Monterrey, Nuevo León 64720; ${ }^{3}$ Medicina Diagnostica, Monterrey; ${ }^{4}$ Department of Immunology, Faculty of Medicine, Autonomous University of Nuevo León, Monterrey, Nuevo León 64460, Mexico

Received March 28, 2013; Accepted May 14, 2013

DOI: $10.3892 /$ br.2013.125

\begin{abstract}
The adipokine leptin plays a critical role in the regulation of reproductive function and there has been growing interest in its potential role in the development of cancers in which obesity is an established risk factor. Serum leptin levels were found to be higher in patients diagnosed with endometrial and ovarian cancer compared to those observed in healthy individuals. This study was conducted to determine the expression of the leptin receptor $(\mathrm{Ob}-\mathrm{R})$ in endometrial biopsies of patients diagnosed with endometrial and ovarian cancer. In this preliminary study, immunohistochemistry (IHC) and the color deconvolution method were used to assess the expression levels of the Ob-R protein in three groups of endometrial tissue: one from patients diagnosed with endometrioid endometrial carcinoma, one from patients diagnosed with ovarian cancer and one from individuals without any diagnosed gynecologic disease (control group). Our results demonstrated that the highest expression of $\mathrm{Ob}-\mathrm{R}$ protein in endometrial biopsies was detected in the ovarian cancer group $(\mathrm{P}=0.000)$. This finding suggests that changes in Ob-R expression may be assessed through the measurement of the optical density of endometrial biopsies and may become a useful tool in preventive screening, particularly for ovarian cancer.
\end{abstract}

Correspondence to: Dr Manuel López-Cabanillas-Lomelí, Faculty of Public Health and Nutrition, Autonomous University of Nuevo León, Ave. Dr Eduardo Aguirre Pequeño y Yuriria, Col. Mitras Centro, Monterrey, Nuevo León 64460, Mexico

E-mail: manuel.lopezc@uanl.mx

Key words: endometrium, endometrial cancer, immunohistochemistry, leptin receptor, ovarian cancer

\section{Introduction}

Gynecologic cancers are responsible for $\leq 11 \%$ of cancer-related mortality cases (1). Obesity has been associated with a 2- to 5-fold increase of the relative risk of developing endometrial cancer. Moreover, in developed countries, this association is considered responsible for $60 \%$ of the incidence of this type of cancer (2-7). Among women who had never used hormone-replacement therapy, obese women, particularly those with a body mass index of $>35.0$, had a 1.5 - to 2 -fold increase in the relative risk of developing ovarian cancer compared to those of normal weight (5). Among obese populations, high serum estrogen levels are considered the main cause of the observed increase in the relative risk of developing endometrial and ovarian cancer $(3,8,9)$. However, the molecular mechanism underlying this association has not been fully elucidated.

Recently, there has been growing interest in the potential role of adipokines in the development of cancers for which obesity is an established risk factor (10). The adipokine leptin is produced in higher quantities in white adipose tissue in vivo and its concentration is elevated in obese patients. In addition, leptin plays a critical role in the regulation of reproductive function (11-13); thus, the investigation of its function in gynecologic malignancies is critical. In addition, serum leptin levels are higher in patients diagnosed with endometrial and ovarian cancer compared to those observed in healthy individuals (14-16). Furthermore, in vitro studies on human cell lines of ovarian and endometrial cancer indicate that leptin leads to the activation of signaling pathways, such as JAK2/STAT3, MAPK/ERK and PI3K/AKT, which stimulate cell proliferation and inhibit apoptosis $(17,18)$, demonstrating its biological ability to promote the malignant behavior of cancers by stimulating growth, migration and invasiveness of tumor cells (19). Those effects are exerted via its binding to its protein receptor (Ob-R), which belongs to the class I cytokine receptor family (20).

In endometrial cancer, immunohistochemistry (IHC) revealed a decreased expression of $\mathrm{Ob}-\mathrm{R}$ compared to normal 
tissue and its expression was higher in well-differentiated compared to poorly differentiated tumors, indicating an association with histological degree $(21,22)$.

By contrast, Ob-R overexpression in ovarian cancer may be a prognostic factor of poor survival (18). Furthermore, evidence suggests that certain ovarian cancers develop from endometrial tissue and the analysis of overexpressed proteins in ovarian cancer should take into consideration the endometrial normal tissue (23). Moreover, the limited success of ovarian cancer screening promotes the development of novel approaches to screening.

Considering the hypothetical role of this protein in the etiopathogenic process of endometrial and ovarian malignancies $(17,18,22)$ and that the theoretically common origin of the two resides in endometrial tissue (23), the aim of this preliminary study was to investigate whether the changes in the Ob-R expression reported in cancer tissues may be visualized in endometrial biopsies and therefore serve a potential biomarker for these two diseases.

\section{Materials and methods}

Study population. A total of 39 endometrial tissue biopsies were obtained from the archives of the Departments of Pathology, Medical Unit of High Specialty, Hospital No. 25 and Gynecology and Obstetrics, Hospital No. 23, Mexican Social Security Institute, Monterrey, Nuevo León, Mexico. The tissues were fixed in $4 \%$ formalin and embedded in paraffin immediately after the biopsy procedure and were performed by physician's request. Cases that had not previously undergone chemotherapy, radiotherapy or endocrine therapy prior to biopsy were selected from the period between 2004 and 2006. The specimens were evaluated by an expert pathologist who reviewed the corresponding slides stained with hematoxylin and eosin, to confirm histological diagnosis $(24,25)$ and select a representative endometrial tissue sample for the IHC assay.

Immunohistochemical detection of $O b-R$. Sections $(4 \mu \mathrm{m})$ were cut from the paraffin blocks and placed on silanized slides (S3002; Dako North America, Carpinteria, CA, USA). Subsequently, slides were immersed twice in xylol for $10 \mathrm{~min}$ per immersion, hydrated in a descending series of ethanol solutions and washed with distilled water. Antigen retrieval was performed by heating the samples for $20 \mathrm{~min}$ at $96^{\circ} \mathrm{C}$ in a retrieval solution (S1699; Dako North America), followed by incubation at room temperature for $20 \mathrm{~min}$. The slides were then washed in buffer (S1968; Dako North America) for 5 min, followed by blocking of endogenous peroxidase (S2001; Dako North America) for $15 \mathrm{~min}$ and incubation in a solution to reduce non-specific binding (X0909; Dako North America) for $10 \mathrm{~min}$. Tissue sections were incubated for $20 \mathrm{~h}$ at $4^{\circ} \mathrm{C}$ in a humidification chamber with an anti-Ob-R primary antibody (sc-80255; Santa Cruz Biotechnology, Santa Cruz, CA, USA), diluted at 1:150. As a negative control, the primary antibody was replaced with $1 \%$ albumin in phosphate-buffered saline. Breast cancer tissue highly expressing Ob-R (histopathological score, $3+$ ) was used as the positive control in each assay. Samples were then incubated for $30 \mathrm{~min}$ with the biotinylated universal link antibody (K0690; Dako North America) as the secondary antibody, followed by a 5-min washing step and incubation with streptavidin-horseradish peroxidase (K0690; Dako North America) for $30 \mathrm{~min}$ at room temperature. The specific reactivity was visualized by incubation with 3,3'-diaminobenzidine tetrahydrochloride (DAB) (K3468; Dako North America) for $10 \mathrm{~min}$, followed by an increase of the intensity of the DAB staining via a 2-min immersion in $0.5 \% \mathrm{CuSO}_{4}$. The slides were contrasted with hematoxylin (S3309; Dako North America) for $1 \mathrm{~min}$, followed by a dehydration step in an ethanol series, clearance in xylene and mounting with a synthetic resin (HX787224; Merck, Darmstadt, Germany).

Histopathological and digital scoring. The histopathological scoring was performed by a pathologist through examination under a light microscope; the staining intensity was classified as follows: 0 , negative $\mathrm{Ob}-\mathrm{R}$ immunostaining; $1+$, weak expression; $2+$, moderate expression; and $3+$, strong expression. To estimate the digital score, all the slides were visualized under an Olympus BX41 microscope (Olympus America, Inc., Melville, NY, USA), magnification, x320. Images were captured with a Nikon D40 camera (Nikon, Inc., Melville, NY, USA) adapted to the microscope using a 10x Carl Zeiss phototube (Carl Zeiss, Thornwood, NY, USA). The format of the JPEG image obtained was RGB color with a 24-bit depth and a resolution of $3008 \times 2000 \mathrm{dpi}$. The background was adjusted using the rolling ball algorithm of Image J software $(\mathrm{NIH}$, $\mathrm{v} 1.45 \mathrm{p}$ ), followed by the extraction of the regions of interest from these images, which was the glandular component in each of the slides. The color deconvolution algorithm was then used to separate the contribution of the DAB and hematoxylin dyes: the image obtained for DAB was analyzed to obtain the median optical density (MOD) value, which ranged from 0 to 255 (black to white) (26). Using the digital score, the antigen content of tissues increased as the detected MOD decreased within the range of 0-255 (black to white) $(25,27)$.

Statistical analyses. The sample size necessary to conduct this study was obtained using Minitab statistical software (version 15.0). The parameters used were as follows: $\alpha=0.05$, $1-\beta=0.95$ and total deviation $(\sigma)=15,913$. The database was captured in the SPSS statistical package (version 20). An association was determined between the expression of Ob-R, evaluated by histopathological score $(0,1+$ and $2+)$, and the three study groups (control, endometrioid endometrial carcinoma and ovarian cancer) via a 3x3 analysis using the Fisher's exact test. In this analysis, the $3+$ score was discarded, as it was not observed in any of the samples. For the quantitative analysis of the variation in the expression of $\mathrm{Ob}-\mathrm{R}$ according to digital score (MOD) in the three groups (control, endometrioid endometrial carcinoma and ovarian cancer), the following tests were applied: one-way analysis of variance (ANOVA), Levene's test (for the comparison of variances) and the Student-Newman-Keuls test in cases for which significance was detected in the means of the groups. $\mathrm{P}<0.05$ was considered to indicate a statistically significant difference.

\section{Results}

$I H C$ and digital score assessment of $O b-R$ expression. The clinical records of the 39 patients enrolled in this study included histological diagnosis and clinicopathological 
Table I. Clinicopathological characteristics of study groups.

\begin{tabular}{|c|c|c|c|}
\hline \multirow[b]{2}{*}{ Characteristic } & \multicolumn{3}{|c|}{ Group } \\
\hline & $\mathrm{CG}(\mathrm{n}=16)$ & OG $(n=12)$ & $\mathrm{EG}(\mathrm{n}=11)$ \\
\hline \multicolumn{4}{|l|}{ Histological diagnosis of biopsies } \\
\hline Atrophic endometrium & 10 & 11 & - \\
\hline Proliferative endometrium & 4 & - & - \\
\hline Secretory endometrium & 2 & 1 & - \\
\hline Endometrioid cancer & - & - & 11 \\
\hline \multicolumn{4}{|l|}{ Age of patients (years) } \\
\hline$<50$ & 4 & 1 & 2 \\
\hline $50-60$ & 6 & 6 & 3 \\
\hline$>60$ & 6 & 5 & 6 \\
\hline \multicolumn{4}{|l|}{ Menopausal status } \\
\hline Premenopausal & 6 & 1 & 1 \\
\hline Postmenopausal & 10 & 11 & 10 \\
\hline \multicolumn{4}{|l|}{ Body mass index } \\
\hline$\leq 24.9$ & 4 & 3 & - \\
\hline $25.0-29.9$ & 10 & 4 & 2 \\
\hline$\geq 30$ & 2 & 5 & 9 \\
\hline \multicolumn{4}{|l|}{ TNM stage } \\
\hline I & - & 4 & 8 \\
\hline II & - & 1 & 1 \\
\hline III & - & 3 & 2 \\
\hline IV & - & 4 & - \\
\hline \multicolumn{4}{|l|}{ Histological grade } \\
\hline G1, well-differentiated & - & 1 & 6 \\
\hline G2, moderately differentiated & - & 5 & 5 \\
\hline G3, poorly differentiated & - & 6 & - \\
\hline
\end{tabular}

CG, control group; OG, ovarian cancer group; EG, endometrial cancer group; TNM, tumor-node-metastasis staging system.

characteristics (Table I). The IHC study of Ob-R expression by histopathological score revealed that the localization of the protein in the glandular epithelium of the endometrium was in the form of fine granules located in the cytoplasm of normal and cancerous glands, presenting occasional nuclear reactivity. Among the control group tissues, 56\% exhibited negative immunostaining for Ob-R (Fig. 1A), whereas the remaining $44 \%$ had a score of $1+$ (Fig. 1B), observed mainly in the cytoplasm of glandular and surface epithelium. Among the ovarian cancer group, $25 \%$ exhibited negative immunostaining for $\mathrm{Ob}-\mathrm{R}$ and $75 \%$ had a score of $1+$ (Fig. 1C) with the same pattern, with occasional reactivity observed in the stroma. Among the endometrioid endometrial carcinoma samples, $36 \%$ exhibited negative immunostaining, $55 \%$ had a score of $1+$ (Fig. 1D) and 9\% had a score of 2+. The histopathological score did not demonstrate a significant association between the expression of $\mathrm{Ob}-\mathrm{R}$ and the three investigated groups $(\mathrm{P}=4.929)$ (Table II). However, digital scoring revealed that the ovarian cancer group expressed significantly higher levels of the $\mathrm{Ob}-\mathrm{R}$ protein $(\mathrm{P}=0.000)$ compared to the other two groups, with an average MOD of 188.25 \pm 14.5 (Table III).
Table II. Association of expression of Ob-R and study groups by histopathological score.

\begin{tabular}{lccc}
\hline Group & 0 & $1+$ & $2+$ \\
\hline Control $(\mathrm{n}=16)$ & $9(56 \%)$ & $7(44 \%)$ & $0(0 \%)$ \\
Ovarian cancer $(\mathrm{n}=12)$ & $3(25 \%)$ & $9(75 \%)$ & $0(0 \%)$ \\
Endometrial cancer $(\mathrm{n}=11)$ & $4(36 \%)$ & $6(55 \%)$ & $1(9 \%)$ \\
aP-value & & $\mathrm{NS}$ & \\
\end{tabular}

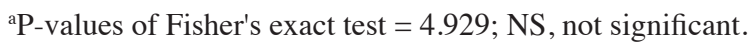

\section{Discussion}

IHC demonstrated that $\mathrm{Ob}-\mathrm{R}$ expression was located mainly in the cell membrane of the endometrial glandular epithelium obtained from biopsies of patients with endometrioid endometrial carcinoma and ovarian cancer (without pathological changes of the endometrium). This finding was consistent with 
A

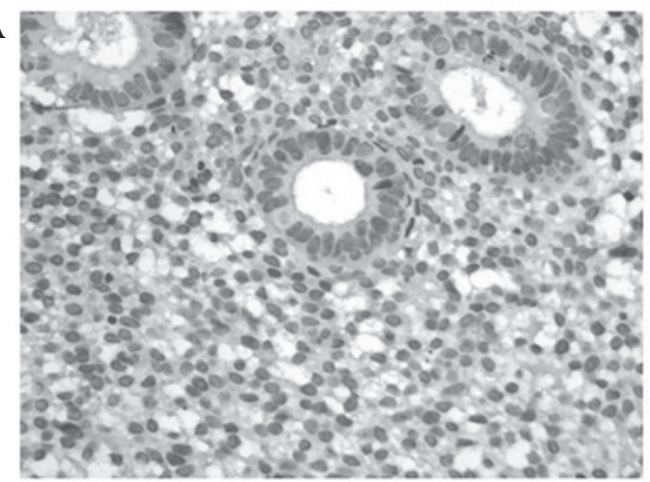

C

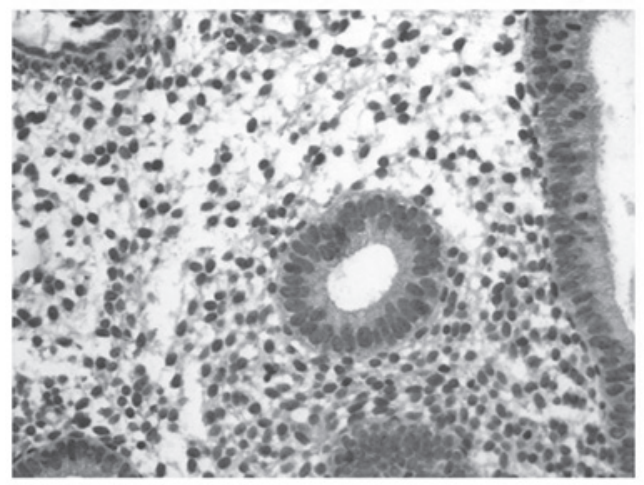

B

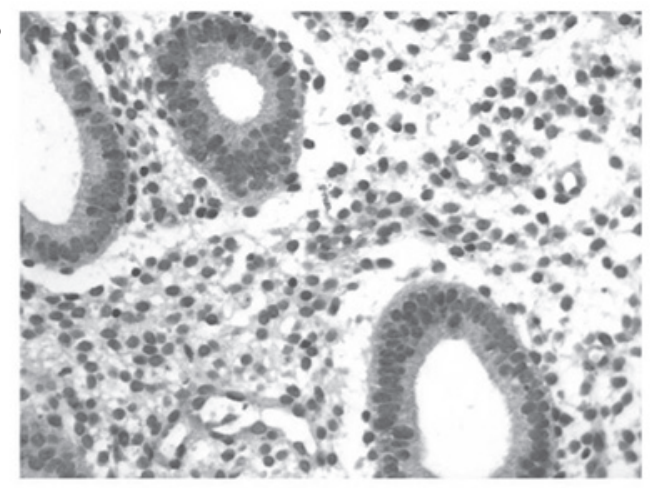

D

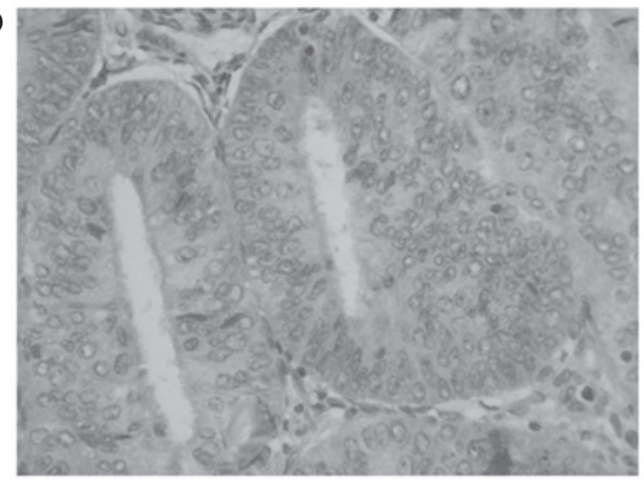

Figure 1. Immunohistochemistry images for Ob-R protein in endometrial tissue: (A) control (negative immunostaining); (B) control (score +1); (C) ovarian cancer; (D) endometrioid endometrial carcinoma. Magnification, x40.

Table III. Mean comparison of Ob-R expression in study groups by digital score.

\begin{tabular}{lcc}
\hline Groups & Mean of MOD \pm SD & aP-value \\
\hline Control $(\mathrm{n}=16)$ & $212.69 \pm 9.9$ & $\mathrm{NS}$ \\
Ovarian cancer $(\mathrm{n}=12)$ & $188.25 \pm 14.5$ & 0.000 \\
Endometrial cancer $(\mathrm{n}=11)$ & $212.82 \pm 9.0$ & $\mathrm{NS}$ \\
\hline
\end{tabular}

MOD, median optical density; SD, standard deviation; ${ }^{\mathrm{a}} \mathrm{P}$-values determined by ANOVA-Student-Newman-Keuls; NS, not significant.

the results of earlier studies $(21,22,28,29)$. However, occasional nuclear reactivity was also detected, which was mentioned only in one previous study on endometrial tissues (28).

Among the control group samples, $44 \%$ were negative and $56 \%$ were positive for $\mathrm{Ob}-\mathrm{R}$ expression, with a histology of atrophic endometrium in 10, proliferative endometrium in 4 and secretory endometrium in 2 cases. This finding was inconsistent with the results of a previous study, according to which $100 \%$ of the samples of histologically normal endometria were negative for $\mathrm{Ob}-\mathrm{R}$ expression (28). This disparity may be partly attributed to the fact that the tissues used in that study were obtained during the mid-to-late proliferative phase of the menstrual cycle, whereas it has been reported that the expression of Ob-R reaches its peak levels during the early secretory phase (30). Moreover, the average age of the 25 patients sampled in that study was 40 years (28), whereas the average age in our control group was 57 years and in the endometrioid endometrial carcinoma and ovarian cancer groups it was 55 and 58 years, respectively, thus exhibiting similar histological characteristics $(24,25)$.

Among our samples of endometrioid endometrial carcinoma, $61 \%$ exhibited positive $\mathrm{Ob}-\mathrm{R}$ reactivity, which was higher compared to that reported by two previous studies, according to which 31 and $47 \%$ of the endometrioid tumors investigated were positive for this protein $(21,29)$. The enhanced expression observed in our study may be the result of the better fixation of tissues achieved with biopsies compared to surgical samples and by the use of DAB enhancer $(31,32)$. Statistical comparison between the levels of expression of $\mathrm{Ob}-\mathrm{R}$ in the groups and the data obtained based on histopathological scores revealed an absence of significant differences. As regards the control and endometrioid endometrial carcinoma groups, despite the lack of significant differences in Ob-R expression, as previously described $(22,28)$, a reduced $\mathrm{Ob}-\mathrm{R}$ expression was observed with loss of differentiation in the endometrial tissue.

Digital scoring revealed that the ovarian cancer group exhibited a significantly higher expression of the Ob-R protein compared to the other two groups. This is consistent with the previously reported advantages of the digitalization of IHC images, particularly regarding quantitative aspects (32-35) and seems to support the theory of ovarian carcinogenesis, which states that certain ovarian cancers are derived from endometrial epithelia (23). In this sample, biopsies of the endometria of patients diagnosed with ovarian cancer also exhibited increased $\mathrm{Ob}-\mathrm{R}$ expression, as was previously observed in ovarian cancer tissues (18).

In conclusion, it was determined that endometrial biopsies exhibit the same tendency of Ob-R expression as that observed in surgical tissue samples. In addition, there was an apparent enhanced expression of $\mathrm{Ob}-\mathrm{R}$ in ovarian cancer patients 
compared to patients diagnosed with endometrial cancer. This finding appears to support the common origin of the two malignancies in endometrial tissue, which may prove useful as a novel screening method for ovarian cancer detection. Additional studies are required to validate our results.

\section{Acknowledgements}

The authors would like to thank Manuel Vera and Ricardo Cerda for their technical assistance and guidance.

\section{References}

1. American Cancer Society: Cancer Facts \& Figures 2011. Atlanta: American Cancer Society, 2011.

2. Bergstrom A, Pisani P, Tenet V, Wolk A and Adami HO Overweight as an avoidable cause of cancer in Europe. Int $\mathrm{J}$ Cancer 91: 421-430, 2001.

3. Calle EE and Kaaks R: Overweight, obesity and cancer: epidemiological evidence and proposed mechanisms. Nat Rev Cancer 4 579-591, 2004

4. Farrow DC, Weiss NS, Lyon JL and Daling JR: Association of obesity and ovarian cancer in a case-control study. Am J Epidemiol 129: 1300-1304, 1989.

5. Leitzmann MF, Koebnick C, Danforth KN, et al: Body mass index and risk of ovarian cancer. Cancer 115: 812-822, 2009.

6. Levi F, La Vecchia C, Negri E, Parazzini F and Franceschi S: Body mass at different ages and subsequent endometrial cancer risk. Int J Cancer 50: 567-571, 1992.

7. Lew EA and Garfinkel L: Variations in mortality by weight among 750,000 men and women. J Chronic Dis 32: 563-576, 1979

8. Lukanova A, Lundin E, Micheli A, et al: Circulating levels of sex steroid hormones and risk of endometrial cancer in postmenopausal women. Int J Cancer 108: 425-432, 2004.

9. Risch HA: Hormonal etiology of epithelial ovarian cancer, with a hypothesis concerning the role of androgens and progesterone. J Natl Cancer Inst 90: 1774-1786, 1998.

10. Vona-Davis L and Rose DP: Adipokines as endocrine, paracrine, and autocrine factors in breast cancer risk and progression. Endocr Relat Cancer 14: 189-206, 2007.

11. Gonzalez RR, Simon C, Caballero-Campo P, et al: Leptin and reproduction. Hum Reprod Update 6: 290-300, 2000.

12. Greisen S, Ledet T, Moller N, et al: Effects of leptin on basal and FSH stimulated steroidogenesis in human granulosa luteal cells. Acta Obstet Gynecol Scand 79: 931-935, 2000.

13. Hamann A and Matthaei S: Regulation of energy balance by leptin. Exp Clin Endocrinol Diabetes 104: 293-300, 1996.

14. Ashizawa N, Yahata T, Quan J, et al: Serum leptin-adiponectin ratio and endometrial cancer risk in postmenopausal female subjects. Gynecol Oncol 119: 65-69, 2010.

15. Cymbaluk A, Chudecka-Glaz A and Rzepka-Gorska I: Leptin levels in serum depending on body mass index in patients with endometrial hyperplasia and cancer. Eur J Obstet Gynecol Reprod Biol 136: 74-77, 2008.

16. Mor G, Visintin I, Lai Y, et al: Serum protein markers for early detection of ovarian cancer. Proc Natl Acad Sci USA 102: 7677-7682, 2005.

17. Gao J, Tian J, Lv Y, et al: Leptin induces functional activation of cyclooxygenase-2 through JAK2/STAT3, MAPK/ERK, and PI3K/AKT pathways in human endometrial cancer cells. Cancer Sci 100: 389-395, 2009
18. Uddin S, Bu R, Ahmed M, et al: Overexpression of leptin receptor predicts an unfavorable outcome in Middle Eastern ovarian cancer. Mol Cancer 8: 74, 2009.

19. Lang K and Ratke J: Leptin and adiponectin: new players in the field of tumor cell and leukocyte migration. Cell Commun Signal 7: 27, 2009

20. Bjorbaek C, Uotani S, da Silva B and Flier JS: Divergent signaling capacities of the long and short isoforms of the leptin receptor. J Biol Chem 272: 32686-32695, 1997.

21. Bogusiewicz M, Semczuk A, Gogacz M, et al: Lack of correlation between leptin receptor expression and PI3-K/Akt signaling pathway proteins immunostaining in endometrioid-type endometrial carcinomas. Cancer Lett 238: 61-68, 2006.

22. Yuan SS, Tsai KB, Chung YF, et al: Aberrant expression and possible involvement of the leptin receptor in endometrial cancer. Gynecol Oncol 92: 769-775, 2004.

23. Kurman RJ and Shih IeM: The origin and pathogenesis of epithelial ovarian cancer: a proposed unifying theory. Am J Surg Pathol 34: 433-443, 2010.

24. Noyes RW, Hertig AT and Rock J: Dating the endometrial biopsy. Am J Obstet Gynecol 122: 262-263, 1975.

25. Silverberg SG, Mutter GL, Kurman RJ, Kubik-Huch RA, Nogales $\mathrm{F}$ and Tavassoli FA: Tumors of the uterine corpus: epithelial tumors and related lesions. In: WHO Classification of Tumors: Pathology and Genetics of Tumors of the Breast and Female Genital Organs. Tavassoli FA and Devilee P (eds). IARC Press, Lyon, pp221-232, 2003.

26. Ruifrok AC and Johnston DA: Quantification of histochemical staining by color deconvolution. Anal Quant Cytol Histol 23: 291-299, 2001.

27. Cornish TC and Haluska MK: Color deconvolution for the analysis of tissue microarrays. Anal Quant Cytol Histol 31: 304-312, 2009.

28. Koda M, Sulkowska M, Wincewicz A, et al: Expression of leptin, leptin receptor, and hypoxia-inducible factor 1 alpha in human endometrial cancer. Ann NY Acad Sci 1095: 90-98, 2007.

29. Wincewicz A, Koda M, Sulkowska M, Kanczuga-Koda L and Sulkowski S: Comparison of STAT3 with HIF-1alpha, Ob and ObR expressions in human endometrioid adenocarcinomas. Tissue Cell 40: 405-410, 2008.

30. Kitawaki J, Koshiba H, Ishihara H, et al: Expression of leptin receptor in human endometrium and fluctuation during the menstrual cycle. J Clin Endocrinol Metab 85: 1946-1950, 2000.

31. Johnson CW: Issue in immunohistochemistry. Toxicol Pathol 27: 246-248, 1999.

32. Cross SS: Observer accuracy in estimating proportions in images: implications for the semiquantitative assessment of staining reactions and a proposal for a new system. J Clin Pathol 54: 385-390, 2001.

33. Diaz LK and Sneige N: Estrogen receptor analysis for breast cancer: current issues and keys to increasing testing accuracy. Adv Anat Pathol 12: 10-19, 2005.

34. Lehr HA, Jacobs TW, Yaziji H, Schnitt SJ and Gown AM: Quantitative evaluation of HER-2/neu status in breast cancer by fluorescence in situ hybridization and by immunohistochemistry with image analysis. Am J Clin Pathol 115: 814-822, 2001.

35. Umemura $\mathrm{S}$, Itoh J, Itoh $\mathrm{H}$, et al: Immunohistochemical evaluation of hormone receptors in breast cancer: which scoring system is suitable for highly sensitive procedures? Appl Immunohistochem Mol Morphol 12: 8-13, 2004 